\title{
Die Entwicklung eines Technologie Radars für den IT-Mittelstand
}

\author{
Marko Berndt und Dana Mietzner*
}

\section{Zusammenfassung}

Die systematische und kontinuierliche Auseinandersetzung mit neuen digitalen Technologien und das frühzeitige Erkennen von Chancen und Risiken als Grundlage für strategische Entscheidungen im Sinne einer langfristigen Aufrechterhaltung der Wettbewerbsfähigkeit, stellt für KMU generell aber auch für mittelständische IT-Unternehmen eine Herausforderung dar. Ursache dafür sind eine hohe Dynamik in den Unternehmensumfeldern, geringe Ressourcen für eine systematische strategische Vorausschau sowie ein Mangel an passfähigen methodischen Ansätzen. Im Mittelpunkt dieses Beitrages steht das Technologie Scouting als ein ausgewählter methodischer Ansatz, um die strategische Vorausschau von KMU der IT-Wirtschaft zu unterstützen. In diesem Beitrag wird gezeigt, wie das Technologie Scouting schlank und agil für eine ausgewählte Branche, den IT-Mittelstand, gestaltet werden kann und wie die Ergebnisse des Scoutings in einem Technologie Radar visualisiert und nutzbar gemacht werden können. Zu diesem Zweck wird im Beitrag die Betaversion eines Technologie Radars vorgestellt.

\section{Abstract}

The systematic and continuous evaluation of new digital technologies and the early recognition of opportunities and risks as a basis for strategic decisions in the sense of maintaining competitiveness in the long term pose a challenge for SME in general, but also for medium-sized IT companies. This is due to the high dynamics in the business environment, low resources for systematic strategic foresight and a lack of suitable methodological approaches. The focus of this paper is technology scouting as a selected methodological approach to support strategic foresight of SME in the IT sector. This article will show how technology scouting can be made lean and agile for a selected IT mid-sized sector, and how the results of scouting can be visualized and harnessed in a technology radar. For this purpose, the article presents the beta version of the technology radar.

\section{Hintergrund und Zielsetzung}

Der Mittelstand wird in zahlreichen Studien und Publikationen immer wieder als Motor oder gar als das Rückgrat der deutschen Wirtschaft bezeichnet (vgl. IfM Bonn 2016, BMWi 2017a). Die aktuellen Ergebnisse des Mittelstandsbarometers von Ernst \& Young verweisen auf eine gute wirtschaftliche Situation im deutschen Mittelstand. Trotz der Zuversicht und einer positiven Auftragslage werden Gefahren wie der Fachkräftemangel oder der zunehmende Wettbewerb nicht außer Acht gelassen (vgl. E\&Y 2018). Die omnipräsente Herausforderung der Digitalisierung schwebt zusätzlich als bedeutender Trend über der deutschen KMU Landschaft. Die mit der Digitalisierung einhergehenden Veränderungen schaffen dabei nicht nur Gewinner. Neue digitale Technologien ermöglichen neue Geschäftsmodelle mit einem disruptiven Charakter. Dadurch geraten bestehende Unternehmen zunehmend unter Druck und ganze Branchen können grundlegend verändert werden. Ein Beispiel für diese Entwicklung ist z. B. das Geschäftsmodell von Twitter, das die Form der Erstellung, Aufbereitung und Verbreitung von Nachrichten fundamental verändert und etablierte Marktakteure unter Druck gesetzt hat. Dabei hat Twitter keine eigene neue Technologie entwickeln müssen, sondern hat verfügbare und sich schnell entwickelnde Technologieplattformen genutzt (vgl. Downes \& Nunes 2013: 48).
Nokia übersah wiederum den Trend des Smartphones und Kodak fand keinen Anschluss an die digitale Fotografie. Beide Unternehmen kostete dies ihre Marktführerschaft (vgl. Schallmo et al. 2017: 289-290, Streibich 2016: 212) Diese Beispiele verweisen darauf, dass es nötig ist, frühzeitig Chancen und Trends zu identifizieren, wie den Einsatz neuer Technologien (vgl. Streibich 2016: 212). Dies stellt jedoch für ein einzelnes Unternehmen eine große Herausforderung dar und ist vor allem für kleine und mittlere Unternehmen unter Umständen schwierig umzusetzen. In der deutschen Unternehmenslandschaft ergibt es Sinn, dass diese Aufgabe im Mittelstand selbst verortet wird, speziell im IT-Mittelstand, der sich an der Schnittstelle 
zwischen IT und deren wirtschaftlicher Entfaltungsmöglichkeit befindet (vgl. Bär et al. 2018: 128). Der IT-Mittelstand besitzt damit im gegenwärtig dominierenden Trend der Digitalisierung von Wirtschaft und Gesellschaft eine Enablerfunktion. Für dessen KMU ist es deshalb erforderlich, sich frühzeitig mit neuen digitalen Technologien auseinanderzusetzen und deren Potenziale zu bewerten. Ein Schlüsselaspekt dieser Aufgabe kann das Technologie Scouting sein. Es ist wichtig, den ITMittelstand nicht nur für das Thema zu sensibilisieren, sondern auch dazu zu befähigen, die Entwicklungsdynamiken von Technologien zu nutzen, um nicht wie Nokia und Kodak von neu auf den Markt drängenden Innovatoren supprimiert zu werden. Dieser Beitrag widmet sich der Frage, wie neue Technologien erkannt, bewertet und systematisch in operative Aktivitäten von Unternehmen überführt werden können.

Dazu wird in dem vorliegenden Beitrag zuerst der IT-Mittelstand vorgestellt. Im Anschluss zeigt dieser Beitrag die Ziele und einen möglichen Prozess des Technologie Scouting auf und präsentiert abschließend eine Möglichkeit zur Visualisierung der Ergebnisse in Form eines online zur Verfügung stehenden Technologie Radars. Der gesamte Prozess wird dabei im Hinblick auf Adaption durch den IT-Mittelstand beleuchtet.

\section{Der IT-Mittelstand und die Rolle von Netzwerken}

Nach der Definition des Instituts für Mittelstandsforschung gehören in Deutschland 9.852 IT-Unternehmen zum ITMittelstand. Berücksichtigt wurden hierbei Unternehmen mit einer Anzahl zwischen 10 und 499 Beschäftigten und einem maximalen jährlichen Umsatz von 50 Millionen Euro (vgl. IfM Bonn 2018). Im Jahr 2015 erwirtschafteten die im deutschen Unternehmensregister eingetragenen IT-Mittelständler 68 Milliarden Euro, was 35 Prozent des gesamten Branchenumsatzes entspricht. Die übrigen 65 Prozent entfallen dabei zu 13 Prozent auf Unternehmen mit 9 oder weniger Beschäftigten und zu ca. 52 Prozent auf die 0,2 Prozent der Unternehmen mit mehr als 500 Angestellten.
Dass knapp 56 Prozent der sozialversicherungspflichtigen Mitarbeiter/innen dabei im IT-Mittelstand arbeiten, verdeutlicht ebenfalls die bereits angedeutete Bedeutung des IT-Mittelstands für die Digitalisierung des Mittelstands insgesamt (vgl. Bitkom e.V. 2018). Im IT-Mittelstand konvergieren eine gewisse Flexibilität, die der agileren, weniger komplexen Unternehmensstruktur geschuldet ist und eine ausreichende Marktpräsenz, um dem Mittelstand insgesamt neue Impulse zu geben. Eben diese Schlüsselposition soll den IT-Mittelstand zum Wegbereiter der Digitalisierung und zum zentralen Treiber der digitalen Transformation werden lassen (vgl. BMWi 2017: 90). Vorschub leisten diesem Ziel eine Vielzahl von IT-Netzwerk-Organisationen in Deutschland. Eine Kienbaum Studie im Auftrag des Bundesministeriums für Wirtschaft und Energie beschreibt, dass diese Netzwerke breit auf regionaler Ebene aufgestellt sind und dass je Bundesland mindestens eine Netzwerk-Organisation besteht. Darunter zählen unter anderem Cluster, Wirtschaftsförderungen, Unternehmensverbände und wissenschaftliche Einrichtungen (vgl. Kienbaum 2016: 26). Die systematische Auseinandersetzung mit neuen Technologien, die Bewertung ihrer Potenziale und die damit einhergehende Schaffung einer Wissensbasis als Grundlage für Strategieprozesse der Unternehmen, kann für einzelne KMU des IT-Mittelstandes, aufgrund von Ressourcenengpässen oder durch den Mangel an passfähigen Methoden, eine enorme Herausforderung darstellen. Eine Möglichkeit die notwendige systematische Auseinandersetzung mit neuen Technologien zu unterstützen, ist die Umsetzung von Ansätzen der strategischen Vorausschau auf der Ebene von branchenspezifischen Netzwerken. Eine ausgewählte Methode der strategischen Vorausschau ist das Technologie Scouting. Das Technologie Scouting ist ein mehrgliedriger Prozess, der bereits einiges an wissenschaftlicher Aufmerksamkeit erfahren hat, dessen Umsetzung in der Wirtschaft allerdings eher noch punktuell anstatt in der Breite stattfindet. Im Folgenden wird daher zuerst das Technologie Scouting vorgestellt und dann detailliert ein methodischer Ansatz skizziert, der Anwendung im IT-Mittelstand finden kann.

\section{Technologie Scouting}

Die eingangs genannten Beispiele früherer Marktführer zeigen die möglichen Auswirkungen der Entwicklungsdynamiken von Technologien auf Unternehmen. Ein Beispiel für die rasante Veränderung bietet die Musikindustrie. Vor knapp 15 Jahren revolutionierte Apple mit dem iTunes Store die Branche. Doch Streamingdienste wie Spotify oder Deezer verdrängen das ehemalige Erfolgsmodell in die Bedeutungslosigkeit (vgl. Châlons \& Duft 2016: 20). Dies ist nur ein Beispiel dafür, dass Technologieentwicklungen einen wesentlichen Treiber für neue Produkte, Dienstleistungen, Prozesse oder Geschäftsmodelle darstellen können. Die Umsetzbarkeit von neuen Geschäftsideen kann durch die Entwicklung einer oder mehrerer Technologien erst ermöglicht werden (vgl. Schimpf et al. 2016: 32). Auf einzelne Unternehmen bezogen liegt die Herausforderung nun darin, Technologieentwicklungen möglichst frühzeitig zu erkennen und daraus entstehende Innovationschancen und -risiken für das eigene Unternehmen zu bewerten. Schimpf et al. stellen fest, dass diese Beobachtung und Bewertung in nahezu jedem Unternehmen stattfindet, jedoch auch, dass ab einer bestimmten Unternehmensgröße oder technologischen Komplexität die Herausforderung besteht, die Identifikation von Technologieentwicklungen abzustimmen, zu koordinieren und Transparenz über die Bewertung zu schaffen. Genau an dieser Stelle setzt das Technologie Scouting an, das Rohrbeck wie folgt definiert: “[...]a systematic approach by companies whereby they assign part of their staff or employ external consultants to gather information in the field of science and technology and through which they facilitate or execute technology sourcing. Technology scouting is either directed at a specific technological area or undirected, identifying relevant developments in technological white spaces. Technology scouting relies on formal and informal information sources, including the personal networks of the scouts" (Rohrbeck 2010: 3). Die Grundidee dabei ist es, ein Netzwerk aus Expertinnen und Experten, bestehend aus den sogenannten Technologie Scouts (die interne oder 
externe Mitarbeiter/innen sein können) aufzubauen, das neue Technologien identifiziert, untersucht und zugänglich macht (vgl. Auth et al. 2017: 940). In der betriebswirtschaftlichen Literatur finden sich viele weitere Begriffe, wie Technology Monitoring, Technology Scanning, Technologiefrühaufklärung oder Technology Intelligence. Schimpf et al. stellen fest, dass die Ansätze der unterschiedlichen Begrifflichkeiten zum Teil mit dem Technologie Scouting gleichgesetzt werden können (vgl. Schimpf \& Lang-Koetz 2010: 9). Dully beschreibt, dass die Grundstruktur des Prozesses in allen Ausprägungen ähnlich ist: vereinfacht gesagt folgt der Erfassung der Informationen die Verarbeitung, bevor die Weiterleitung an die Empfänger erfolgt (vgl. Dully 2011: 42). Rohrbeck spricht dabei von einer Trichterlogik (siehe Abb. 1), die Technologien von der Identifizierung bis zur Umsetzung durchlaufen. Schrittweise verringern sich dabei die als relevant eingestuften Technologien, bis am Ende eine bestimmte Auswahl von einsetzbaren Technologien übrigbleibt (vgl. Auth et al. 2017: 941). Eine übliche Visualisierung der Ergebnisse des Technologie Scouting erfolgt oft durch ein sogenanntes Technologie Radar.

Detaillierter betrachtet teilt Rohrbeck den Technologie Scouting Prozess in vier Phasen ein: (1) Die Identifikationsphase, (2) die Selektionsphase, (3) die Bewertungsphase und (4) die Verbreitungsphase (vgl. Rohrbeck 2007: 6). Schimpf et al. verfolgen einen ähnlichen Ansatz. Die im Folgenden vorgestellten Phasen stellen eine Synthese der Aspekte beider Quellen dar und dienen als Grundlage für die Planung eines Technologie Scouting Prozesses für den IT-Mittelstand.

\subsection{Die Identifikationsphase}

In der ersten Phase sollten zunächst für das Unternehmen relevante Technologien und Anwendungsfelder abgegrenzt werden, um den Prozess möglichst zielgerichtet und effektiv zu gestalten. Grundlagen dafür stellen beispielsweise im Unternehmen vorhandene Kompetenzen und technologische Möglichkeiten dar, aktuelle und zukünftig erforderliche Funktionalitäten bestehender Anwen-

dungen oder technologische, gesellschaftliche, ökonomischen bzw. ökologische Trends. Schimpf et al. konstatieren, dass die Identifikation von Technologie- und Anwendungsfeldern in starker Abhängigkeit zur Technologiestrategie des Unternehmens steht. Die Schwierigkeit besteht darin, dass zukünftige technologische Entwicklungen in der Strategie berücksichtigt werden müssen (vgl. Schimpf et al. 2010: 10).

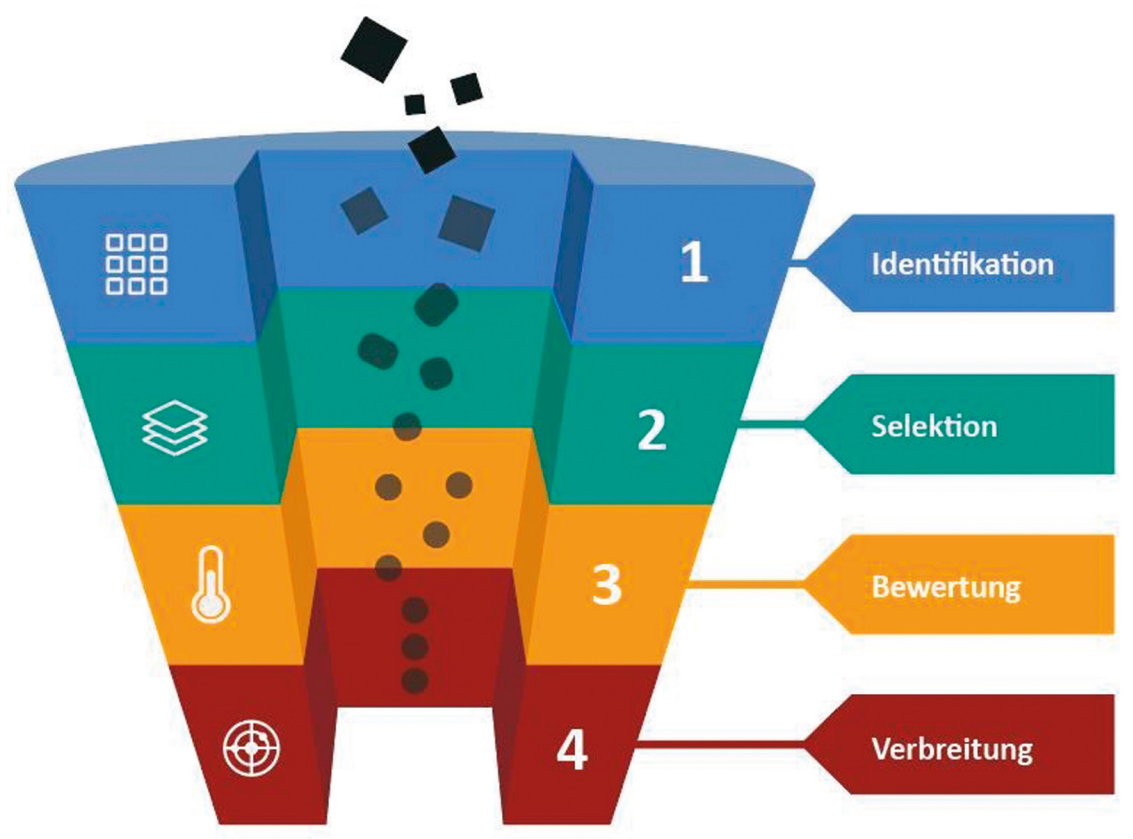

Abb. 1) Der Technologie Scouting Prozess. Eigene Darstellung in Anlehnung an Rohrbeck 2007: 8.

\subsection{Die Selektionsphase}

In der zweiten Phase werden zu den identifizierten Technologien Informationen gesammelt. Einerseits können diese Informationen aus internen Quellen bezogen werden, sofern Expertinnen und Experten für eben jene Entwicklungen im Unternehmen beschäftigt sind (zum Beispiel im Rahmen von F\&E). Allerdings ist in den meisten Fällen davon auszugehen, dass die strukturierte Einbindung externer Informationsquellen unumgänglich ist. Die Vielzahl und Mannigfaltigkeit von Angeboten in diesem Sektor stellt die Unternehmen vor die Aufgabe, eine sinnvolle Auswahl und darüber hinaus eine sinnvolle Verknüpfung vorzunehmen. Neben formalen Quellen, wie Studien, Geschäftsberichten oder Patentdatenbanken existieren auch infor- melle Quellen, wie Gesprächsrunden von Expert/innen, öffentliche Förderdatenbanken oder persönliche Kontakte (vgl. Schimpf \& Lang-Koetz 2010: 10, Rohrbeck 2007: 5). Qualität und Informationsdichte variieren je nach Quelle. Weiterhin stellt die Informationsbeschaffung oftmals einen nicht unerheblichen Kosten- und Zeitfaktor für die Unternehmen dar (vgl. Dully 2011: 45). 
Die eigentliche Beurteilung der ermittelten Technologien sollte im Abgleich mit der Technologiestrategie des Unternehmens vorgenommen werden. Dabei können verschiedene Methoden zum Einsatz kommen (beispielsweise Markt- und Patentanalysen, Befragungen oder Delphi Studien). Die Wahl der Methode hängt dabei von der Verfügbarkeit von Ressourcen ab und es gilt, dass sie den entsprechenden Anforderungen des Einzelfalls angepasst werden kann (vgl. Schimpf \& Lang-Koetz 2010: 11).

\subsection{Die Verbreitungsphase}

In der vierten Phase erfolgt die Kommunikation der Ergebnisse. Sie stellt einen wesentlichen Erfolgsfaktor für den Gesamtprozess dar, da die gewonnenen Erkenntnisse nur dann Wirkung entfalten können, wenn sie entsprechend kommuniziert werden und den richtigen Akteurinnen und Akteuren zur Verfügung stehen. Nicht nur die Managementebene, sondern auch Beschäftigte mit technischem Schwerpunkt sollten dabei einbezogen werden (vgl. Schimpf \& Lang-Koetz 2010: 11). Endprodukte dieser Phase können Berichte, Dokumentationen, OnlineWikis oder eben ein Technologie Radar sein (vgl. Rohrbeck 2007: 7).

\section{Technologie Radar}

Ein Technologie Radar bietet den Vorteil einer grafischen Visualisierung, die neben der Verortung der Technologien eine kurze Zusammenfassung inklusive aktueller Entwicklungen, des Forschungsstatus und des wirtschaftlichen Potentials beinhaltet. Als Basis der Darstellung dient dabei oft das eponyme Radar, das heißt eine Unterteilung eines Kreises in Quadranten und kreisförmige Distanzlinien (Ringe). Quadranten stellen dabei üblicherweise nominale Attribute dar (z. B. einen Technologiesektor), Ringe stehen für die Metrik, die in Phase (3) zur Beurteilung verwendet wurde (z.B. Erzielung der Marktreife in ein, zwei oder drei Jahren). In der Praxis existiert eine Vielzahl von spezifischen Visualisierungen (siehe Abb. 2) für verschiedenste Zwecke (vgl. Rohrbeck 2007: 7).

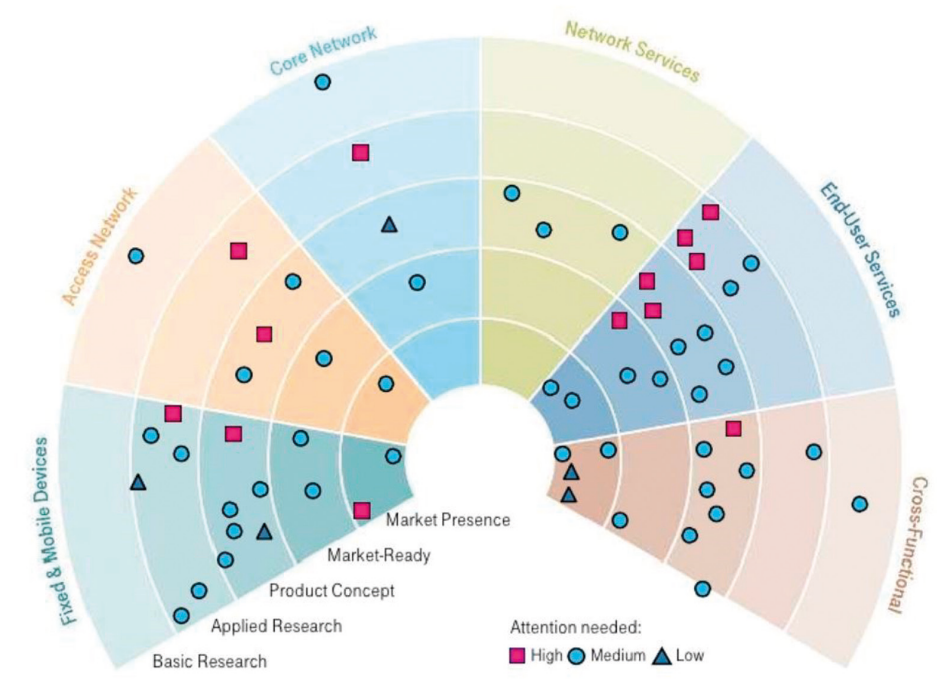

Abb. 2) Beispiel Technologie Radar der Deutschen Telekom (Hartenthaler 2009: 10).

Nachdem es erstmalig 2004 bei der Deutschen Telekom eingesetzt wurde, hält das Technologie Radar mittlerweile sukzessive Einzug in die deutsche Unternehmerlandschaft (vgl. Rohrbeck 2017: 6). Neben der Deutschen Telekom nutzen es beispielsweise der Online-Versandhändler Zalando und der Online-Marktplatz AutoScout24 (vgl. Zalando 2018, AutoScout24 2018). Üblicherweise erfolgt die Klassifizierung der Technologien unter Einbeziehung der Relevanz für das Geschäftsmodell des Unternehmens und des Reifegrads der Technologie (vgl. Golovatchev et al. 2008: 993). Zu den Funktionen des Technologie Radars gehören (1) die Frühwarnung und -aufklärung möglicher Gefahren aus dem Unternehmensumfeld aus technologischer Sicht, (2) die Identifikation potenzieller neuer Wettbewerber, die dieselbe Technologie anbieten, jedoch ein erweitertes Funktionsprofil aufweisen, (3) die Sicherung von Planungszyklen und die flexiblere Reaktionsfähigkeit auf technologische Veränderungen und (4) die Identifikation von strategischen Fenstern, bei der zum Beispiel die eigene Technologie mit einer weiteren kombiniert werden kann, um neue Märkte zu adressieren (vgl. Ardilio 2012: 61f.). Der Einsatz eines Technologie Radars ermöglicht zusammengefasst die strukturierte Beobachtung technologischer Entwicklungen und deren frühzeitige Einbindung in die Unternehmensplanung. Weiterhin wird eine Grundlage für die Kommunikation geschaffen, durch die verschiedene Hand- lungsoptionen berücksichtigt werden können (vgl. Schimpf et al. 2016: 42). Die Bewertung einer Vielzahl komplexer Technologien kann in der Form eines Technologie Radars verdichtet und greifbar präsentiert werden (vgl. Auth et al. 2017: 946).

\section{Zwischenfazit - Technologie Scouting im IT-Mittelstand}

Zwar zeigen die eingangs vorgestellten Beispiele von Nokia und Kodak, dass in den wettbewerbsintensiven Märkten, auf denen auch der IT-Mittelstand agiert, Technologie Scouting als Geschäftsnotwendigkeit verstanden werden muss (vgl. Golavtchev \& Budde 2010: 760), jedoch ist diese Notwendigkeit selten unmittelbar. In Anbetracht der Tatsache, dass die vollständige Umsetzung des geschilderten Prozesses für ein einzelnes Unternehmen einen erheblichen personellen und finanziellen Aufwand bedeutet, verknüpft mit positiver Auftragslage und dem Problem des Personalmangels, kann deshalb davon ausgegangen werden, dass das Technologie Scouting einen eher geringen Stellenwert im IT-Mittelstand aufweist. Aus diesem Grund erscheint eine geeignete Anpassung des dargestellten Prozesses für die vom Mittelstand geprägte IT-Wirtschaft sinnvoll, um einen niederschwelligen Einstieg in das Thema zu ermöglichen. Ziel ist es dabei nicht nur für das Technologie Scouting zu sensibilisieren, sondern die Unternehmen zu befähigen, die Methode selbst anzuwenden. 


\section{Ein vereinfachter Prozess für den IT-Mittelstand}

Die vier Phasen des beschriebenen Technologie Scouting Prozesses bedeuten einen hohen Zeitaufwand, wenn sie von Grund auf neu umgesetzt werden. Dieser lässt sich jedoch erheblich reduzieren, wenn auf bereits vorhandenes Wissen zurückgegriffen werden kann. Eine Reduzierung der Granularität, zum Beispiel von spezifischen Technologien hin zu Einflussfaktoren, verringert die erforderlichen Ressourcen, beispielsweise den personellen Aufwand. Für die Phasen (1) und (2) wurde im konkreten Beispiel daher auf die Trend-Aggregationswebseite TRENDEXPLORER von TRENDONE zurückgegriffen. Die Bewertung in Phase (3) erfolgte an Hand des Technology Readiness Level (siehe Abb. 3), ebenfalls unter Reduzierung der Komplexität von neun auf drei Stufen. Für Phase (4) wurde eine Open Source Bibliothek unter Visualisierungs-, Effizienz-, Skalierungs- und Datenschutzgesichtspunkten angepasst. Die folgenden Abschnitte detaillieren diese Schritte.

Phasen (1) und (2):

Ermittlung der Inhalte

Bereits einfache Internetrecherchen liefern Technologie Radare, die aktuelle, relevante und fundierte Daten visualisieren (vgl. Thoughtworks 2018, Zalando 2018, AutoScout24 2018). Innerhalb des Mittelstand 4.0-Kompetenzzentrum Netzwerkes in Deutschland existiert ebenfalls ein aktuelles Technologie Radar, das kostenfrei zugänglich ist (vgl. Kompetenzzentrum Hamburg 2018). Um den Zielen der Aufwandsreduktion und Generalisierung Rechnung zu tragen, wurde kein weiteres Technologie Radar mit spezifischen Technologien entwickelt, sondern vielmehr ein Technologie Radar erstellt, das spezifische Einflussbereiche zusammenfasst und an Hand von Signalen Beispiele zur Anwendung aufkommender oder aktueller Einflussfaktoren zeigt. Signale sind hier Zeichen aus der Wissenschaft und Wirtschaft, die auf neue Anwendungen von Technologien im IT-Bereich verweisen (vgl. IFTF 2018). Das hier vorgestellte Vorgehen stellt daher eher ein ergänzendes denn ein substituierendes Angebot der bereits verfügbaren Radare dar.
Wie bereits erwähnt, ist die Sichtung, Bewertung und Aufbereitung von Technologie Trends häufig aufwendig und kostenintensiv. Mittlerweile gibt es jedoch einige Scouting Plattformen, die es sich zur Aufgabe gemacht haben, diesen Prozess zu automatisieren und aus verschiedenen Quellen weltweit Trends zu identifizieren und in einer Datenbank zusammenzuführen (vgl. Mapegy 2018, Trendone 2018). Als Quellen dienen dabei unter anderem Pressemitteilungen, Forschungsberichte oder Geschäftsberichte. TRENDONE verfolgt beispielsweise den Anspruch, Trends innerhalb von 30 Sekunden zu erschließen (vgl. Trendone 2018). TRENDONE teilt zur Übersicht Trends in Mega-, Makro- und Micro-Trends ein (siehe Abb. 3). Von Bedeutung ist dabei die automatische, hierarchische Kategorisierung der ermittelten Daten. So kann auf jeder Hierarchieebene auf Statistiken zurückgegriffen werden, die eine Einschätzung der Bedeutung des jeweiligen Trends für eine ausgewählte Branche erlauben. quellen. Diese Art der Informationsermittlung ist daher ideal für einen ersten Einstieg in die Thematik, ersetzt aber für strategische Unternehmensentwicklung nicht eine fundierte Analyse.

Da die oben beschriebene Datenbank weitgehend automatisiert arbeitet, erscheint es sinnvoll, weitere, nicht technologie-basierte Quellen, heranzuziehen. Dies können beispielsweise Studien oder Expertenmeinungen sein. Für das Technologie Radar für den IT-Mittelstand wurden hierbei unterschiedliche Forschungsarbeiten verwendet, die Technologietrends der Zukunft im IT-Mittelstand untersuchten (vgl. Bitkom e.V. 2018: 19, BMWi 2017b). Die Ergebnisse wurden mit den jeweiligen Trends der Datenbank verglichen und angepasst.

\section{Phase (3) Bewertung der Trends}

Eine Bewertung identifizierter Technologien ist - wie bereits beschrieben - an Hand einer Vielzahl von Metriken mög-

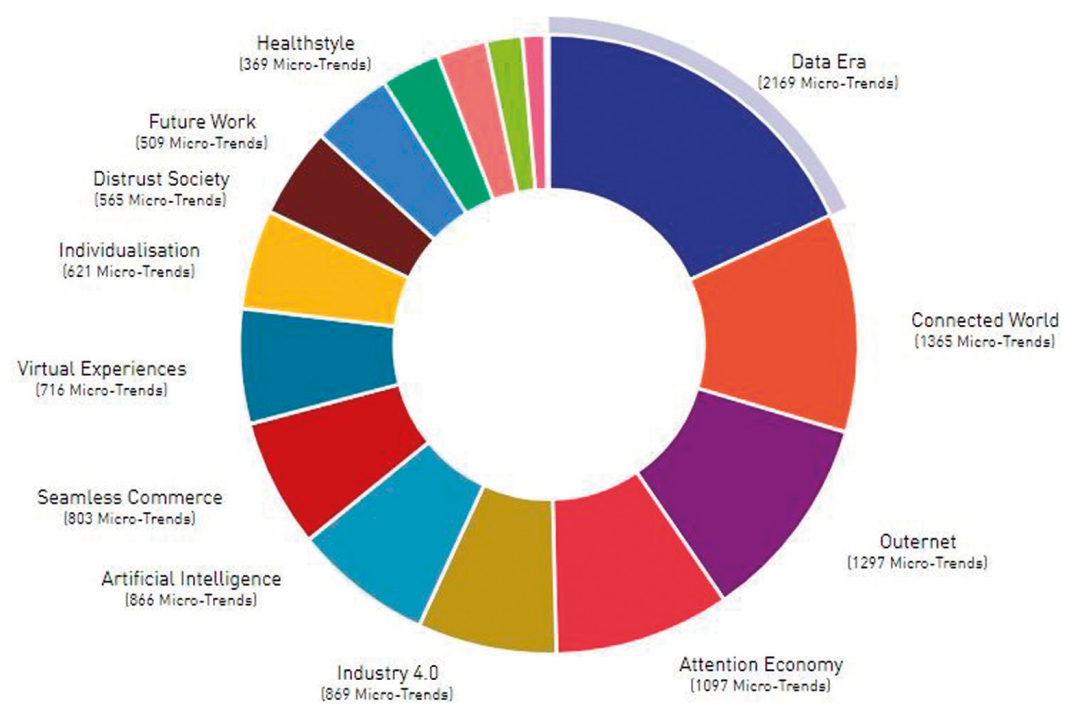

Abb. 3) Trendaggregation des TRENDEXPLORERS (Trendone 2018).

Die Nutzung einer solchen Datenbank als primäre Quelle zur Befüllung des Technologie Radars bietet folgende Vorteile: (1) Automatische Hierarchisierung einschließlich Gewichtung (so lassen sich zum Beispiel die wichtigsten Einflussbereiche leicht identifizieren und dann durch drill down spezifizieren). (2) Vermeidung des größten Anteils an Zeitaufwand, nämlich Beschaffung und Sichtung von Einzel- lich. Auf den ersten Blick sowohl leicht greifbar als auch relativ leicht zu bewerten ist dabei der zeitliche Horizont, innerhalb dessen eine Umsetzung möglich bzw. geboten scheint. Das wohl bekannteste Verfahren zur Bestimmung des Reifegrades ist das TRL-Konzept (Technology Readiness Level), das in den 1980er Jahren von der NASA entwickelt wurde (vgl. Warschat et al. 2015: 59). Der Ansatz eignet sich vor allem, 
um die Marktnähe zu beschreiben (vgl. Preissler 2016: 121). Technologien werden hierbei in neun TRL-Stufen eingeteilt. Diese Stufen reichen von der Beobachtung wissenschaftlicher Grundlagen (TRL-Stufe 1) bis hin zum Einsatz der Technologie im Feld (TRL-Stufe 9). Dabei können drei Stadien eine gröbere Einteilung ermöglichen, die vom Forschungs-, über das Prototypen- bis hin zum Marktreifestadium reichen (siehe Abb. 4).
(4) Visualisierung der gewonnenen Erkenntnisse

Die Visualisierung in Form eines Radars ist mit - je nach gewählter Ausprägung - teils erheblichem Aufwand verbunden. Grundsätzlich existieren dabei verschiedene Möglichkeiten, von rein statischen Darstellungen (beispielsweise als Grafik) bis hin zu voll dynamischen Radaren (Datenbasis editierbar in der Cloud, responsive Oberfläche, etc.). Im

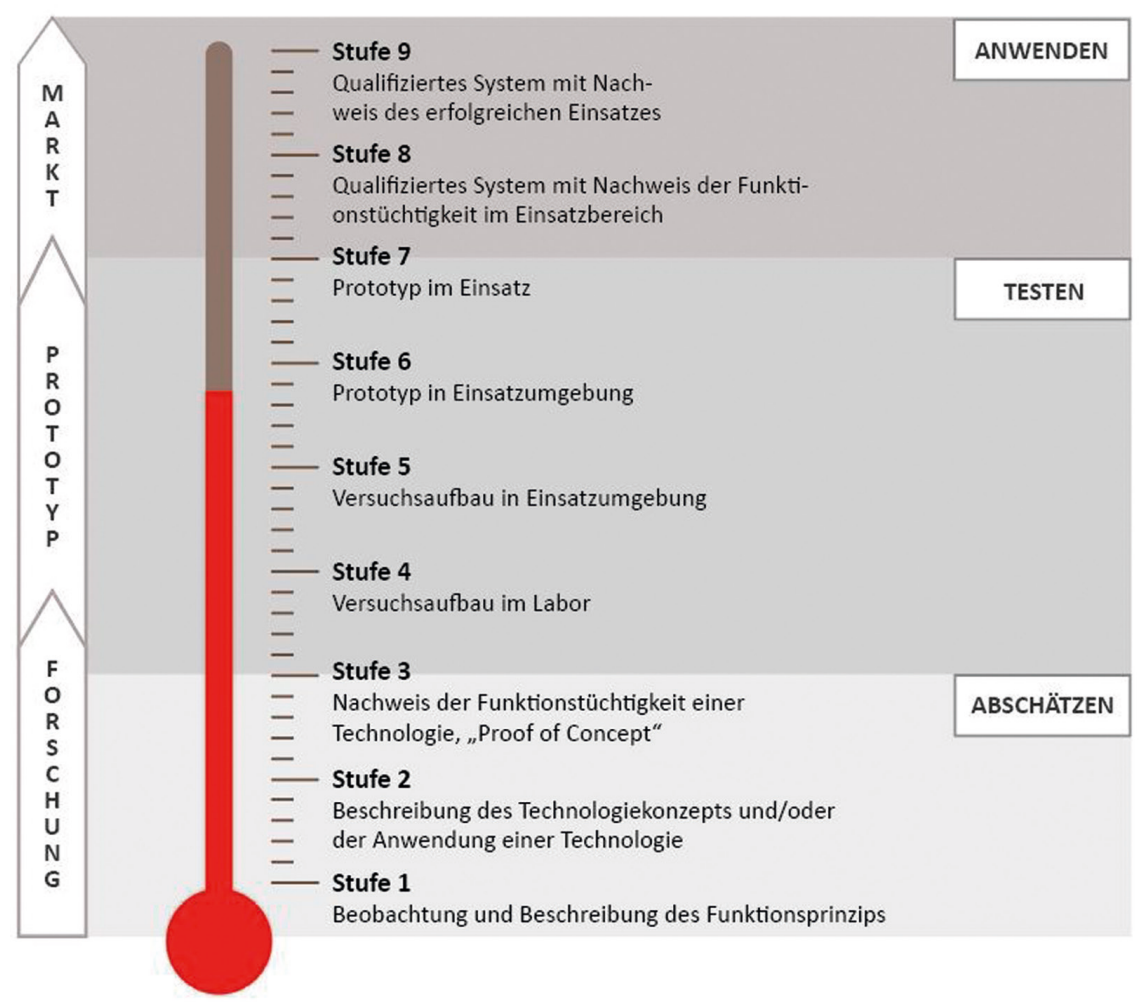

Abb. 4) Technology Readiness Level (Eigene Darstellung in Anlehnung an Mankins 1995).

Für das hier vorgestellte Technologie Radar wurde eben diese reduzierte Granularität gewählt. Durch eine genaue Untersuchung der Quellen der Signale wird dem dazugehörigen Einflussfaktor eines der Stadien zugewiesen. Die drei Stadien werden durch drei Ringe im Radar visualisiert, um Einflussfaktoren grafisch zu verorten. Diese Festlegung ermöglicht eine Umsetzung von Phase (3) mit vertretbarem Aufwand und eignet sich als Einstieg und Grundlage für das Setzen von Impulsen für Innovationsaktivitäten.
Gegensatz zu der Komplexitätsreduktion für die Phasen (1) bis (3) wurde für die Phase (4) eine dynamische und flexible (dadurch entsprechend technisch komplexere) Umsetzung gewählt. Dadurch kann das vorliegende Beispiel sowohl leicht erweitert, als auch leicht auf andere Domänen übertragen werden, das heißt die anfängliche Umsetzungskomplexität fällt bei Transfer zum Beispiel zu interessierten Unternehmen weg und ermöglicht eine sehr einfache Visualisierung von Ergebnissen eines unternehmenseigenen Scouting Prozesses.

\section{Technische Umsetzung des Technologie Radars}

Ein Pionier der digitalen Umsetzung eines Technologie Radars ist das multinationale Softwareunternehmen Thoughtworks. Thoughtworks hat es sich zur Aufgabe gemacht, die Softwarebranche weiterzuentwickeln und erarbeitetes Wissen zu teilen. Aus diesem Grund veröffentlicht Thoughtworks halbjährlich ein Radar mit Technologien, die für die Entwicklung von Software relevant sind (vgl. Thoughtworks 2018). Die hierfür verwendete Softwareplattform steht als Open Source bei Github zur Verfügung und diente als Grundlage für die technische Umsetzung des eigenen Radars (vgl. Github 2018).

Dieser ursprüngliche Code wurde stark erweitert, vor allem in Hinblick auf Skalierbarkeit und Benutzerfreundlichkeit. Ergänzt wurde eine Datenbereitstellung entweder auf Excel-Basis oder über ein Datenbank-Backend, so dass ein Mehrbenutzersystem mit Versionierung ohne Probleme umzusetzen wäre. Aus dieser Datenbasis werden mittels Java automatisiert, sowohl Detailseiten für jeden Einflussfaktor erstellt, als auch die Überblicksvisualisierung in Form des eigentlichen Radars generiert. Des Weiteren wurde die grafische Darstellung überarbeitet. Die Bereitstellung der Web-Anwendung erfolgt über das node.js Framework.

\section{Komponenten der Visualisierung im Technologie Radar}

Die vier Quadranten des Radars stellen Einflussbereiche dar, die als relevant für den IT-Mittelstand identifiziert wurden. Innerhalb dieser Quadranten werden relevante Einflussfaktoren verortet, die wiederum Signale beinhalten (siehe Abb. 5).

Einflussbereiche (A): Die Zusammenstellung der Einflussbereiche teilt das Radar in vier Quadranten auf. Ganz trennscharf sind diese nicht, das bedeutet, dass einige Signale auch anderen Quadranten zugeordnet werden könnten. Dennoch helfen die Quadranten dabei, eine Grundordnung zu schaffen. Die einzelnen Einflussbereiche lassen sich genauer durch einen Klick auf den Auswahlpunkt betrachten. 
Technologiereifegrad (B): Die Ringe des Radars beschreiben den Reifegrad der Einflussfaktoren (C). Gemäß der Metapher des Radars steigt der Reifegrad der Einflussfaktoren mit der Annäherung ans Zentrum. Dabei geben die einzelnen Ringe bereits mögliche Handlungsempfehlungen, die Einflussfaktoren anzuwenden, zu testen oder sie zunächst abzuschätzen.

Signale (D): Die Signale sind den Einflussfaktoren zugeordnet und somit erst in einer tieferen Ebene des Technologie Radars ersichtlich. Signale können Entscheider inspirieren und somit Impulse für eigene Innovationsaktivitäten geben. Durch einen Klick auf den gewünschten Einflussfaktor öffnet sich nicht nur eine Erklärung desselben, sondern zusätzlich ein konkretes Beispiel Signal des Einflussfaktors inklusive der Quelle. Zusätzliche Signale und eine Zusammenfassung der Bewertung sowie die Zuordnung zu einer spezifischen Technologie runden die Zusammenfassung der Einflussfaktoren ab.

Technologie Tags (E): Sind nur einzelne, spezifische Technologien und ihnen zugeordnete Signale von Interesse, kann ein Schieberegler der jeweiligen Technologie aktiviert werden. Dies ermöglicht dem Betrachter eine gezieltere Suche von Einflussfaktoren.
9. Kommunikation der Inhalte des Technologie Radars

Wie bereits ausgeführt, ist die initiale Zielsetzung neben der Bereitstellung von Informationen die Sensibilisierung für die Thematik des Technologie Scouting. Die Ergebnisse sind deshalb als Grundlage für weitere Arbeiten zu verstehen. So soll das Technologie Radar im Laufe der Zeit spezifiziert, vervollständigt und agil weiterentwickelt werden. Es soll Impulse für die unternehmenseigene Technologiestrategie setzen und für aufkommende Technologieentwicklungen sensibilisieren.

\section{Fazit und Ausblick}

Auf Grund der Bedeutung des Mittelstandes für die deutsche Unternehmenslandschaft einerseits und der immer weiterwachsenden Möglichkeiten der digitalen Technologien und dem damit verbundenen Wettbewerbsdruck andererseits, erscheint es unumgänglich, die Digitalisierung des Mittelstands rasch und effizient voranzutreiben, wobei dem IT-Mittelstand eine tragende Funktion zukommt. Technologie Scouting spielt dabei eine wichtige Rolle. Während die Marktführer der IT-Branche die entsprechenden Prozesse bereits fest im Unternehmen verankert haben, sind zwar beim IT-Mittelstand vereinzelte Bemühungen im Bereich der technologischen Vorausschau durchaus festzustellen, jedoch scheint diese in der
Breite noch nicht angekommen sein. Dabei sollte angesichts der besonders hohen Wettbewerbsdynamik das Technologie Scouting als geschäftsnotwendig angesehen werden, um langfristig erfolgreich am Markt agieren und das eigene Unternehmen weiterentwickeln zu können.

Die Betaversion des hier vorgestellten Technologie Radars wird im nächsten Schritt Unternehmen des IT-Mittelstands und Branchenexpertinnen und -experten zum Testen zur Verfügung gestellt. Das Feedback wird aufgenommen, ausgewertet und zur Weiterentwicklung des Radars verwendet. Eine wiederholte Iteration ist denkbar und antizipiert. Als Endprodukt entsteht ein Technologie Radar für den IT-Mittelstand (vgl. http:// radar.itwirtschaft.de/).

Mittelfristig wäre eine offene Plattform zum Crowdsourcing der relevanten Einflussbereiche bzw. Einflussfaktoren denkbar. Incentivierung potentieller Teilnehmer/innen, Güte der Ergebnisse (im Vergleich zu automatisierten Verfahren wie z.B. TRENDONE) und auch der Vergleich mit der Situation und Wahrnehmung von Technologien und entsprechenden Anwendungen in anderen Branchen sowie die Gestaltung der Schnittstellen zu Strategieprozessen sind weitere interessante Forschungsbereiche.

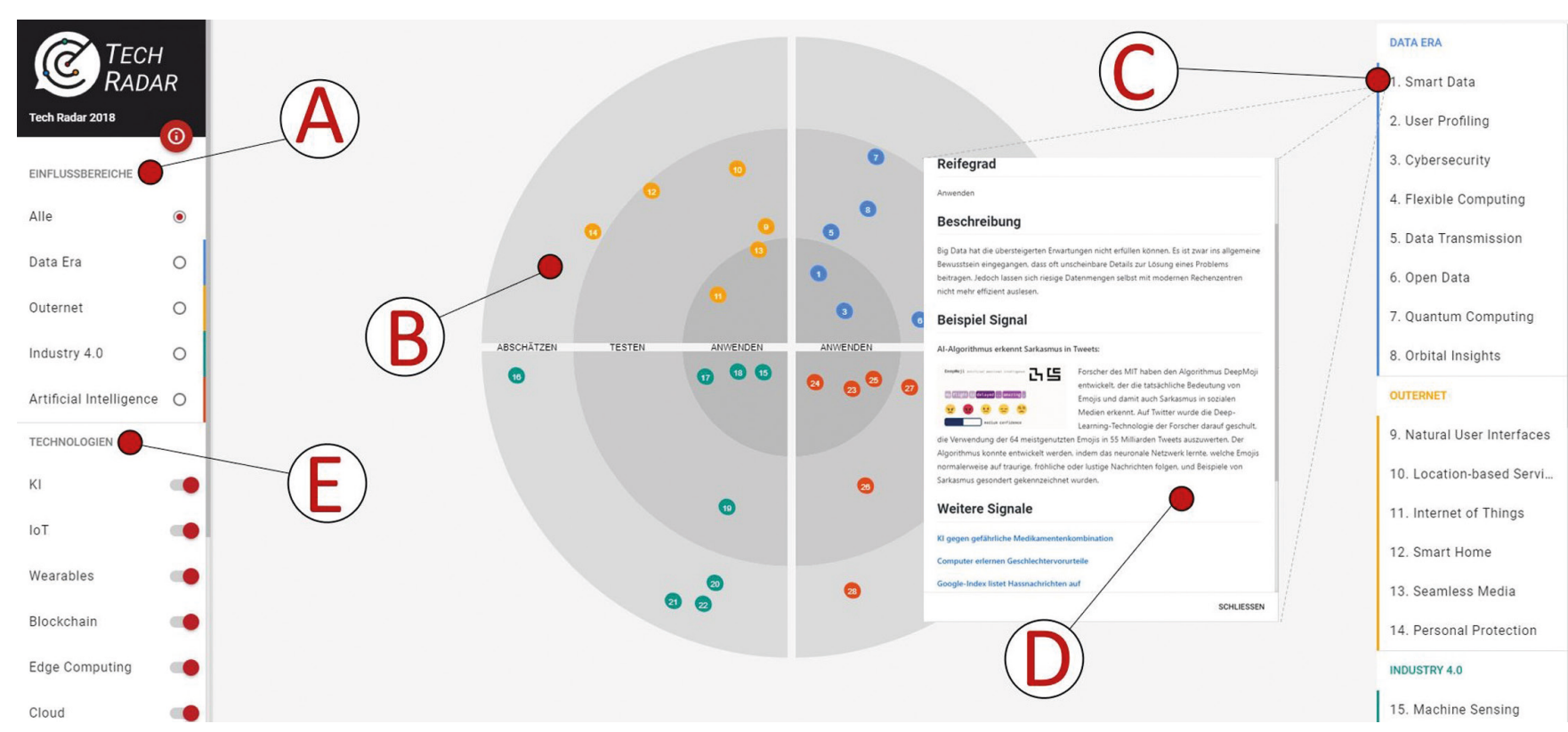

Abb. 5) Beschreibung der einzelnen Komponenten des Technologie Radars (eigene Darstellung). 


\section{LITERATUR}

Ardilio A (2012) Fraunhofer TechnologieRadar: Trends erkennen - Technologien umsetzen. In: Bullinger HJ (ed) Fokus Technologiemarkt. Technologiepotenziale identifizieren - Marktchancen realisieren, Hanser, München, pp 78-90

Auth G, Meyer P, Porst G (2017) Erkennung und Nutzung von Technikinnovationen für den Digital Workplace der Deutschen Telekom. HMD 54(6):935-949. doi: 10.1365/ s40702-017-0365-7

AutoScout24 (2018) Autoscout Radar Feb 2018. https:// radar.thoughtworks.com/?sheetld=https\%3A\%2F\%2Fraw. githubusercontent.com\%2FScout24\%2Fas24-tech-radar\% 2Fmaster\%2Fradars\%2FAutoScout24\%2520Radar\%2520F eb\%25202018\%2520.csv. Accessed 03 Sep 2018

Bär C, Grädler T, Mayr R (eds) (2018) Digitalisierung im Spannungsfeld von Politik, Wirtschaft, Wissenschaft und Recht, 1. Band: Politik und Wirtschaft. Springer Gabler, Berlin. ISBN: 978-3-662-55719-8. doi: 10.1007/978-3-662-55720-4

Bitkom e.V. (2018) Der IT-Mittelstand in Deutschland. Bundesverband Informationswirtschaft. Telekommunikation und neue Medien e. V., Berlin

BMWi (2017a) Wirtschaftsmotor Mittelstand Zahlen und Fakten zu den deutschen KMU. https://www.bmwi.de/ Redaktion/DE/Publikationen/Mittelstand/wirtschaftsmotor-mittelstand-zahlen-und-fakten-zu-den-deutschen-kmu pdf?_blob=publicationFile\&v=1. Accessed 03 Sep 2018

BMWi (2017b) Monitoring-Report Wirtschaft DIGITAL 2017

Châlons C, Dufft N (2016) Die Rolle der IT als Enabler für Digitalisierung. In: Abolhassan F (ed) Was treibt die Digtalisierung? Warum an der Cloud kein Weg vorbeiführt, Springer Gabler, Wiesbaden, pp 27-37

Dully S (2011) Internetbasiertes Technologie-Scouting für Recyclingtechnologien. Dissertation, Universität Stuttgart

Downes, L, Nunes P (2013) Big Bang Disruption. Harvard Business Review: 44-56

EY (2018) Mittelstandsbarometer 2018. https://www. ey.com/Publication/vwLUAssets/ey-mittelstandsbarometer-januar-2018/\$FILE/ey-mittelstandsbarometer-januar-2018.pdf. Accessed 03 Sep 2018

Github (2018) Thoughtworks - build-your-own-radar. https://github.com/thoughtworks/build-your-own-radar. Accessed 03 Sep 2018

Golovatchev | Budde O (2010) Technology and innovation radar - Effective instruments for the development of a sustainable innovation strategy. In: 2010 IEEE Internationa Conference on Management of Innovation \& Technology, 02.06.-05.06.2010, Singapore, Singapore. IEEE, ISBN: 978 1-4244-6565-1, pp 760-764.

doi: 10.1109/ICMIT.2010.5492855

Granig P (2007) Innovationsbewertung, 1. Aufl. Gabler Verlag, s.I. ISBN: 3835007793

Grün, O (2018) Datenöknomie braucht einen offenen Markt. In: Bär C, Grädler T, Mayr R (eds) Digitalisierung im Spannungsfeld von Politik, Wirtschaft, Wissenschaft und Recht, 1. Band: Politik und Wirtschaft. Springer Gabler, Berlin, pp 127-135

Haag C, Schuh G, Kreysa J, Schmelter K (2011) Technologiebewertung. In: Schuh G (ed) Technologiemanagement. Handbuch Produktion und Management 2. 2 ., Springer-Verlag, Berlin Heidelberg, pp 309-366

Hartenthaler J (2009) Vision 2050: Von der physischen Mobilität zur virtuellen Präsenz. https://www.slideshare. net/hartenthaler/vision-2050-5491055. Accessed 03 Sep 2018
IfM Bonn (2016) KMU in Deutschland gemäß der KMU-Definition des IfM Bonn. https://www.ifm-bonn.org/ fileadmin/data/redaktion/statistik/unternehmensbestand/ dokumente/KMU-D_2012-2016_IfM-Def.pdf. Accessed 03 Sep 2018

IfM Bonn (2018) KMU-Definition des IfM Bonn. https:// www.ifm-bonn.org/definitionen/kmu-definition-des-ifmbonn/. Accessed 03 Sep 2018

FTF (2018) Signals. http://www.iftf.org/what-we-do/ foresight-tools/signals/. Accessed 03 Sep 2018

Kienbaum (2016) Machbarkeitsstudie. Vernetzung von mittelständischen IT-Anbieterunter-nehmen in flexiblen Wertschöpfungspartnerschaften über ein IT-Mittelstandszentrum. Kienbaum Management Consultants $\mathrm{GmbH}$, Berlin

Kompetenzzentrum Hamburg (2018) Einführung zum Technologieradar. https://www.kompetenzzentrumhamburg.digital/angebot/technologieradar/einfuehrungzum-technologieradar. Accessed 03 Sep 2018

Kompetenzzentrum IT-Wirtschaft (2018) Wir vernetzen IT made in Germany. https://itwirtschaft.de/. Accessed 03 Sep 2018

Kompetenzzentrum IT-Wirtschaft (2019): Technologie Radar. radar.itwirtschaft.de. Accessed 18 Feb 2019

Mankins JC (1995) Technology Readiness Level. Office of Space Access and Technology NASA

Mapegy (2018) Tap into innovation. https://scout.mapegy.com/. Accessed 03 Sep 2018

Mittelstand-Digital (2017) Zypries gibt Startschuss für drei weitere Mittelstand 4.0-Kompetenzzentren. https://www.mittelstand-digital.de/MD/Redaktion/DE/ Pressemitteilungen/2017/2017-12-04-zypries-gibt-startschuss-fuer-drei-weitere-mittelstand-4-0-kompetenzzentren.html. Accessed 03 Sep 2018

Preissler S (2016) Interorganisationaler Wissens- und Technologietransfer. Dissertation

Rohrbeck R (2007) Technology Scouting - a case study on the Deutsche Telekom Laboratories. ISPIM-Asia 2007 Conference, 12.01.2007 New Dehl

Rohrbeck R (2010) Harnessing a network of experts fo competitive advantage: technology scouting in the ICT industry. R\&D Management 40(2):169-180. doi: 10.1111/j.1467-9310.2010.00601.x

Rummel S (2014) Eine bewertungsbasierte Vorgehensweise zur Tauglichkeitsprüfung von Technologiekonzepten in der Technologieentwicklung. Dissertation, Universität Stuttgart

Schallmo D, Rusnjak A, Anzengruber J, Werani T, Jünge M (eds) (2017) Digitale Transformation von Geschäftsmodellen. Grundlagen, Instrumente und Best Practices. Schwerpunkt. Springer Gabler, Wiesbaden. ISBN: 978-3658-12387-1. doi: 10.1007/978-3-658-12388-8

Schimpf S, Lang-Koetz C (eds.) (2010) Technologiemonitoring. Technologien identifizieren, beobachten und bewerten. Fraunhofer IAO, Stuttgart

Schimpf S, Heubach D, Rummel S (2016) Technologieentwicklung als Innovationstreiber in bestehenden und disruptiven Märkten - von der Beobachtung zur Umsetzung. In: Abele T (ed) Die frühe Phase des Innovationsprozesses, Springer Fachmedien Wiesbaden, Wiesbaden, pp. 31-49

Streibich K-H (2016) Software verändert die Welt: Die Zukunft gehört dem digitalen Unternehmen. In: Bär C, Fischer AT, Gulden H (eds) Informationstechnologien als Wegbereiter für den steuerberatenden Berufsstand. Springer Berlin Heidelberg, Berlin, Heidelberg, ISBN: 978-3-662-44908-0, pp 207-214. doi: 10.1007/978-3-662 44909-7_20

t3n (2018) So eklatant ist der IT-Fachkräftemangel in Deutschland. https://t3n.de/news/it-fachkraeftemangelmint-1078563/. Accessed 03 Sep 2018
Thoughtworks (2018) Technology Radar VOL. 18. Insights into the technology and trends shaping the future. https://www.thoughtworks.com/de/radar. Accessed 03 Sep 2018

Trendone (2018) Trendone - Wir begleiten Sie in die Zukunft. https://www.trendexplorer.com/de/ueber-uns/ ueber-trendone/. Accessed 03 Sep 2018

Warschat J, Schimpf S, Korell M (2015) Technologien frühzeitig erkennen, Nutzenpotenziale systematisch bewerten. Methoden, Organisation, semantische Werkzeuge zur Informationsgewinnung und -speicherung. Ergebnisse des Verbundforschungsprojektes syncTech - synchronisierte Technologieadaption als Treiber der strategischen Produktinnovation. Fraunhofer-Institut für Arbeitswirtschaft und Organisation. Fraunhofer-Verlag: Stuffgart. ISBN 978-38396-0881-4

Zalando (2018) Zalando Tech Radar - 2018.05. https:// opensource.zalando.com/tech-radar/. Accessed 03 Sep 2018

\section{AUTOREN}

Marko Berndt, M.A.

Prof. Dr. rer. pol. Dana Mietzner

Forschungsgruppe Innovations- und Regionalforschung

echnische Hochschule Wildau

E-Mail für Korrespondenz:

dana.mietzner@th-wildau.de

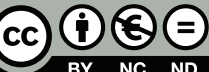

